\title{
HIV-I genotypic drug resistance in patients with virological failure to single-tablet antiretroviral regimens in southern Taiwan
}

This article was published in the following Dove Press journal: Infection and Drug Resistance

\author{
Hung-Chin Tsai ${ }^{1-3}$ \\ I-Tzu Chen' \\ Susan Shin-Jung Lee Le, $^{1,2}$ \\ Yao-Shen Chen ${ }^{1,2}$ \\ 'Division of Infectious Diseases, \\ Department of Medicine, Kaohsiung \\ Veterans General Hospital, Kaohsiung, \\ Taiwan; ${ }^{2}$ Faculty of Medicine, School \\ of Medicine, National Yang-Ming \\ University, Taipei, Taiwan; ${ }^{3}$ Department \\ of Parasitology, Kaohsiung Medical \\ University, Kaohsiung, Taiwan
}

Correspondence: Hung-Chin Tsai; Yao-Shen Chen

Division of Infectious Diseases, Department of Medicine, Kaohsiung Veterans General Hospital, \#386 Ta-Chung Ist Road, Kaohsiung 8I3, Taiwan

Tel +8867342 2I 21 ext 2029

Fax +88673468292

Email hctsail0II@yahoo.com.tw; yschen@vghks.gov.tw
Purpose: Sparse data are available on the prevalence of resistance among HIV-1-infected patients with virological failure to a single-tablet regimen (STR). This study aimed to evaluate the prevalence of HIV genotypic drug resistance in HIV-1-infected patients with virological failure to STRs in southern Taiwan.

Patients and methods: This retrospective study investigated drug resistance in patients with virological failure to STR from January 2016 to September 2017. Antiretroviral resistance mutations were defined using the 2017 International AIDS Society-USA HIV drug resistance algorithm, and drug resistance was compared using the HIVdb program of the Stanford University HIV Drug Resistance Database. Variables between resistance and non-resistance groups were compared.

Results: Thirty-nine HIV-1-infected patients with treatment failure were tested for resistance, of whom $89 \%$ were infected by men who have sex with men. Subtype B HIV-1 strains were found in $90 \%$ of the patients. Eight patients were treatment naïve and initiated STRs, while 31 patients experienced treatment failure after switching to STRs. Eighty-seven percent of the patients harbored any of four classes of resistance (nucleoside reverse transcriptase inhibitors, non-nucleoside reverse transcriptase inhibitors, protease inhibitors (PIs), and integrase strand transfer inhibitors). The prevalence rates of nucleoside reverse transcriptase inhibitor, nonnucleoside reverse transcriptase inhibitor, PI, and integrase strand transfer inhibitor resistance were $72 \%, 82 \%, 10 \%$, and $3 \%$, respectively. Patients with PI resistance were more likely to respond to treatment with a non-tenofovir disoproxil fumarate/emtricitabine/efavirenz-based STR $(P=0.004)$ and a longer duration of antiretroviral therapy (101 months [72.0-123.3] vs 11 months [7-44], $P=0.007$ ). There were no associations between different STRs and transmission risk factors, HIV subtype, duration of antiretroviral therapy, and resistance to tenofovir disoproxil fumarate.

Conclusion: A high rate of antiretroviral drug resistance was found in the patients who failed STR treatment. The presence of PI resistance in these patients represented an inappropriate switch from a multiple tablet regimen to an STR. These findings should remind clinicians that detailed drug resistance history and close monitoring are mandatory after switching to an STR. Keywords: HIV, single-tablet regimen, drug resistance, treatment failure, antiretroviral therapy

\section{Introduction}

Since 1995, the introduction of highly active antiretroviral therapy (HAART) has made HIV infection a manageable disease. ${ }^{1-4}$ However, with the widespread use of antiretroviral therapy (ART), the emergence of drug-resistant HIV could compromise the clinical outcomes in both treatment-naïve and treatment-experienced patients. Transmitted drug resistance to the HIV-1 strain can severely limit the treatment options for new patients 
and shorten the time to treatment failure. ${ }^{5}$ Furthermore, continuing with failed regimens may lead to more complex mutation patterns, the development of cross-resistance to other drugs, and the forward transmission of drug-resistant HIV to ART-naïve patients. ${ }^{6}$ The reported prevalence rates of transmitted drug resistance and acquired ART resistance in Taiwan, where routine viral load monitoring is available, are about $8 \%-10 \%$ and $75 \%$, respectively. ${ }^{7-9}$ As of December 2017, an estimated 29,881 people were living with HIV/AIDS in Taiwan, with most being men who have sex with men (MSM; 62.3\%) and injecting drug users (19.5\%). ${ }^{10}$

A single-tablet regimen (STR) has been associated with better drug adherence ${ }^{11}$ and improved quality of life compared to multiple tablet regimens. ${ }^{12}$ Patients receiving the STR efavirenz/emtricitabine/tenofovir disoproxil fumarate (EFV/ FTC/TDF, Atripla; Gilead Sciences, Foster City, CA, USA) have been reported to have a significantly decreased risk of drug resistance mutations compared to those receiving the same components individually in a non-STR regimen. ${ }^{13}$ In a large French multicenter cohort study, Cotte et a ${ }^{14}$ reported a virological failure rate of $2 \%(10 / 499)$ in patients initially treated with an STR (Atripla/Complera, FTC/rilpivirine/ TDF, FTC/RPV/TDF; Gilead Sciences), which was lower compared to the rate of $5.7 \%(154 / 2,713)$ in patients receiving a non-STR.

TDF/FTC/EFV (Atripla) was first introduced to Taiwan in 2010, followed by TDF/FTC/RPV (Complera) and abacavir $(\mathrm{ABC}) / 3 \mathrm{TC} /$ dolutegravir (DTG) (Triumeq; ViiV Healthcare, Research Triangle Park, NC, USA) in June 2016. The prevalence of HIV-1 drug resistance to these three STRs is unknown. This study aimed to establish the prevalence of genotypic drug resistance (GRT) after failure of STR antiHIV therapy in southern Taiwan, and to establish the clinical and viral characteristics that may be predictive of any drug resistance.

\section{Patients and methods Study design and participants}

This retrospective study was being investigated in southern Taiwan from January 2016 to September 2017. For the purpose of this study, treatment-experienced patients with virological failure were defined as those having an HIV-1 viral load of $\geq 1,000$ copies $/ \mathrm{mL}$. All of the enrolled patients underwent GRT testing. A standardized questionnaire was used to collect demographic data including age, gender, risk factors for HIV infection, CD4 count, viral load, and duration and name of the ART used. First-line ART is provided for free at 69 designated hospitals nationwide by infectious disease physicians and supported by HIV case managers. Due to financial constraints on the provision of free access to combination ART (cART), the Taiwan Centers for Disease Control limits the prescription of cART to antiretroviral-naive HIV-1-infected patients who received their first cART after 1 June 2012. There are currently four STRs approved for use in Taiwan (in order of approval): 1) $\mathrm{TDF} / \mathrm{em}$ tricitabine/EFV (Atripla) in 2010;2) TDF/ emtricitabine/RPV (Complera) in June 2016; 3) dolutegravir/abacavir/lamivudine (Triumeq) in June 2016; and 4) tenofovir alafenamide (TAF)/emtricitabine/elvitegravir/ cobicistat (Genvoya) in September 2017. These four STRs are recommended for the initial therapy in current Taiwan HIV treatment guidelines. Complera is listed as an "alternative" regimen in patients with HIV RNA $<100,000$ copies/ $\mathrm{mL}$ and in patients with a CD 4 count $>200$ cells $/ \mu \mathrm{L}$. Atripla, Complera, and Triumeq were the only three STRs available when this study was conducted. The standard follow-up of HIV-infected patients on ART consisted of out-patient visits every 3 months, with physical examinations, CD4+ T cell count, viral load, hematology, and biochemistry. However, HIV-1 GRT testing for patients with virological failure was not routinely performed. Blood samples for such patients were sent to the Centers for Disease Control for GRT once in every 3 years or to the reference laboratory if the patient signed informed consent. ${ }^{9}$

\section{GRT testing}

Resistance testing for protease/reverse transcriptase (PR/RT) (pol gene) was performed on plasma samples using ViroSeq HIV-1 Genotyping System version 2.8, according to the manufacturer's instructions (Celera, Alameda, CA, USA). Integrase strand-transfer inhibitor (INSTI) resistance was determined using in-house population sequencing. ${ }^{15,16}$ Antiretroviral resistance to PR/RT (pol gene) and to INSTI was interpreted using the HIVdb program of the Stanford University HIV Drug Resistance Database (http://hivdb.stanford.edu; date last accessed 1 Jan 2018). The patients classified as having low-level resistance, intermediate resistance, and high-level resistance were defined as having drug resistance. Resistance-associated mutations were defined according to the presence of at least one mutation included in the 2017 drug resistance mutation list of the International AIDS Society-USA consensus guidelines. ${ }^{17}$

\section{Statistical analysis}

Mann-Whitney U test was used to compare the median values of continuous variables between groups (resistance and wild virus), while Fisher's exact test was used to compare categorical variables between the two groups. A two-sided $P$-value $<0.05$ was considered to be statistically significant. 
Statistical calculations were performed using SPSS version 12.0 (SPSS Inc., Chicago, IL, USA).

\section{Ethical statement}

The study was approved by the institutional review board of Kaohsiung Veterans General Hospital in Taiwan (VGHKS98CT1-08 and VGHKS13-CT4-12). The protocol complied with the ethical considerations involving human subjects, and all information obtained followed standard clinical guidelines. All the study participants provided written informed consent.

\section{Results}

A total of 39 HIV-1 infected STR users who failed treatment and were tested for resistance were enrolled. Among these 39 patients, $94.9 \%(n=37)$ were male and $79.5 \%(n=31)$ were aged 20-39 years. Moreover, 89.5\% (34/38) were MSM. Eight patients were treatment naïve and initiated STR, and 31 patients had failed treatment after switching to an STR. Upon virological failure, the median CD4 cell count was 288 (interquartile range [IQR]: $136-410$ ) cells $/ \mu \mathrm{L}$, with a viral load of $4.7 \log$ (IQR: 3.9-5.1). Most (90\%) of the 39 patients had HIV subtype B (Table 1).

The median duration of HAART was 13 months (IQR: 8-46 months), and the median duration of current HAART upon presentation for GRT was 6 months (IQR: 2-9 months). Upon presentation for GRT, Atripla was used in 28 (71.8\%) patients, Complera in $8(20.5 \%)$ patients, and Triumeq in 3 (7.7\%) patients.

Of the 39 patients with GRT, $87 \%$ had drug resistance to any of the four classes of antiretroviral drugs, including $72 \%$ showing resistance to nucleoside reverse transcriptase inhibitors (NRTIs), $82 \%$ to non-nucleoside reverse transcriptase inhibitors (NNRTIs), 10\% to protease inhibitors (PIs), and $3 \%$ showing resistance to INSTIs (Figure 1). Among the 3 Triumeq users with virological failure, one developed drug resistance to DTG, elvitegravir, and raltegravir (E138K, G140S, Q148H). This patient had ever used raltegravir for 22 months before switching to Triumeq. Among the 36 STR (Atripla/Complera) users with virological failure, none had INSTI resistance.

Only $5 \%$ of the 39 STR users were resistant to zidovudine, $23 \%$ to TDF, and $10 \%$ were resistant to to atazanavir/ritonavir or lopinavir/ritonavir. Of the patients who failed Complera or Triumeq treatment, $38 \%$ and $33 \%$ were resistant to atazanavir/ritonavir or lopinavir/ritonavir, respectively (Figure 2).

Ninety percent of the patients had drug resistanceassociated mutations to any of the four classes of antiretroviral
Table I Demographic data of the 39 HIV-I-infected patients with virological failure to STRs

\begin{tabular}{|c|c|c|}
\hline Parameters & & Number of patients (\%) \\
\hline \multirow[t]{2}{*}{ Gender } & Male & 37 (94.9) \\
\hline & Female & $2(5.1)$ \\
\hline \multirow[t]{4}{*}{ Age (years) } & $20-29$ & $14(35.9)$ \\
\hline & $30-39$ & $17(43.6)$ \\
\hline & $40-49$ & $6(15.4)$ \\
\hline & $>50$ & $2(5.1)$ \\
\hline \multirow[t]{3}{*}{ Risk factor ( $n=38)$} & Heterosexual & $3(7.9)$ \\
\hline & MSM & $34(89.5)$ \\
\hline & IDU & I (2.6) \\
\hline \multirow[t]{2}{*}{ Any class resistance } & Yes & $34(87.2)$ \\
\hline & No & $5(12.8)$ \\
\hline \multirow[t]{2}{*}{ HIV subtype } & Non-B & $4(10.3)$ \\
\hline & B & $35(89.7)$ \\
\hline \multirow[t]{3}{*}{ CD4 (cells $/ \mu \mathrm{L})$} & Minimum & 3 \\
\hline & Maximum & 835 \\
\hline & Median (IQR) & $288(136-4 \mid 0)$ \\
\hline CD4 cut-off point (cells/ & $\geq 200$ & $25(64.1)$ \\
\hline$\mu \mathrm{L})$ & $<200$ & $14(35.9)$ \\
\hline \multirow[t]{3}{*}{ Viral load (log) } & Minimum & 3.30 \\
\hline & Maximum & 5.63 \\
\hline & Median (IQR) & $4.71(3.94-5.12)$ \\
\hline Viral load cut-off point & $\geq 4$ & $29(74.4)$ \\
\hline$(\log )$ & $<4$ & $10(25.6)$ \\
\hline \multirow[t]{2}{*}{ HBV } & Negative & $33(84.6)$ \\
\hline & Positive & $6(15.4)$ \\
\hline \multirow[t]{2}{*}{$\mathrm{HCV}$} & Negative & $39(100)$ \\
\hline & Positive & $0(0)$ \\
\hline \multirow[t]{3}{*}{ Months on HAART } & Minimum & 2 \\
\hline & Maximum & $|3|$ \\
\hline & Median (IQR) & $13(8.0-46.0)$ \\
\hline Months of current & Minimum & 1 \\
\hline \multirow[t]{2}{*}{ regimen } & Maximum & 23 \\
\hline & Median (IQR) & $6(2.0-9.0)$ \\
\hline Months of current & $\geq 6$ & $21(53.8)$ \\
\hline regimen cut-off point & $1-6$ & $18(46.2)$ \\
\hline Months of current & $\geq 12$ & $6(15.4)$ \\
\hline regimen cut-off point & $1-12$ & $33(84.6)$ \\
\hline \multirow[t]{3}{*}{ CR } & Atripla & $28(7 \mid .8)$ \\
\hline & Complera & $8(20.5)$ \\
\hline & Triumeq & $3(7.7)$ \\
\hline \multirow[t]{3}{*}{ IR } & STR & $8(20.5)$ \\
\hline & NNRTI-based & $22(56.4)$ \\
\hline & PI-based & $9(23.1)$ \\
\hline \multirow[t]{4}{*}{ NRTIs in IR } & ZDT/3TC & $22(56.4)$ \\
\hline & TDF/3TC & $2(5.1)$ \\
\hline & TDF/FTC & $3(7.7)$ \\
\hline & Others & $12(30.8)$ \\
\hline \multirow[t]{3}{*}{ NNRTIs in IR (n=22) } & EFV & $16(72.7)$ \\
\hline & NVP & $4(18.2)$ \\
\hline & RPV & $2(9.1)$ \\
\hline \multirow[t]{2}{*}{ Pls in IR (n=9) } & Boosted-PI & $6(66.7)$ \\
\hline & Unboosted-PI & $3(33.3)$ \\
\hline
\end{tabular}

Notes: Pls in IR: boosted-PI consisted of lopinavir/ritonavir $(n=5)$ and atazanavir/ ritonavir $(n=l)$ and unboosted-PI consisted of atazanavir $(n=3)$. Atripla, efavirenz/ emtricitabine/tenofovir disoproxil fumarate; Complera, emtricitabine/rilpivirine/ tenofovir disoproxil fumarate; Triumeq, abacavir/dolutegravir/lamivudine. Abbreviations: $C R$, current regimen; $E F V$, efavirenz; HAART, highly active antiretroviral therapy; HBV, hepatitis B virus; HCV, hepatitis C virus; IDU, intravenous drug user; $I Q R$, interquartile range; IR, initial regimen; MSM, men who have sex with men; NRTI, nucleoside reverse transcriptase inhibitor; NNRTI, non-nucleoside reverse transcriptase inhibitor; NVP, nevirapine; PI, protease inhibitor; RPV, rilpivirine; STR, single-tablet regimen: TDF/3TC, tenofovir disoproxil fumarate/lamivudine; TDF/FTC, tenofovir disoproxil fumarate/emtricitabine; ZDT/3TC, zidovudine/lamivudine. 


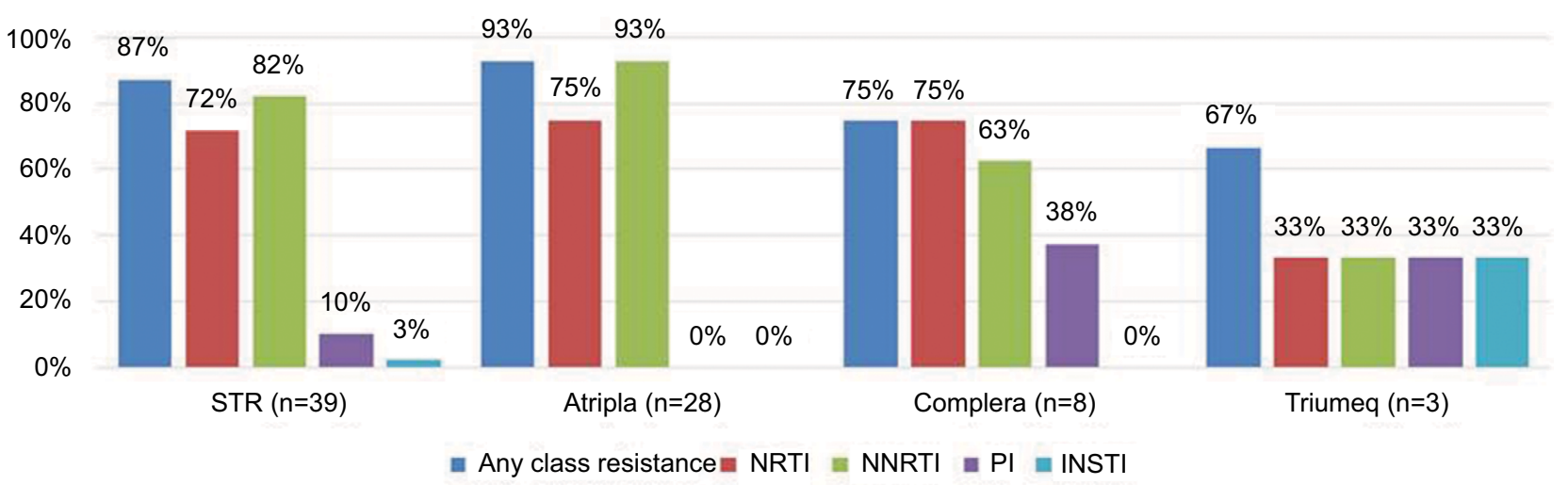

Figure I Drug resistance according to the HIVdb program of Stanford University among 39 HIV-I-infected patients with virological failure to STRs.

Note: The figure shows $87 \%$ drug resistance to any of the four classes of antiretroviral drugs, including $72 \%$ resistance to NRTI, $82 \%$ resistance to NNRTI, $10 \%$ resistance to $\mathrm{PI}$, and $3 \%$ resistance to INSTI.

Abbreviations: INSTI, integrase strand transfer inhibitor; NRTI, nucleoside reverse transcriptase inhibitor; NNRTI, non-nucleoside reverse transcriptase inhibitor; PI, protease inhibitor; STR, single-tablet regimen.
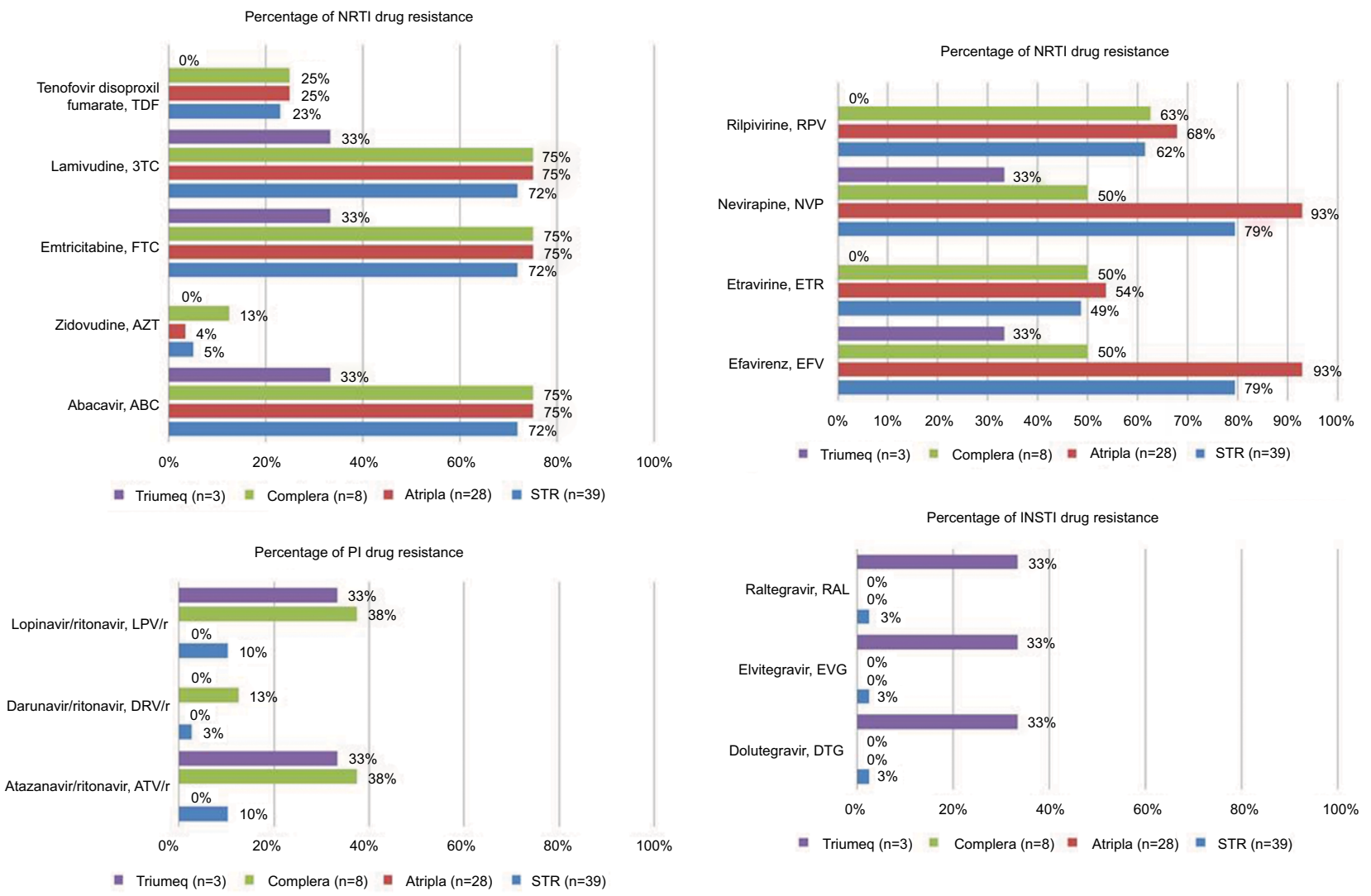

Figure 2 The prevalence of drug resistance to NRTI, NNRTI, PI, and INSTI among 39 HIV-I-infected patients with virological failure to STRs.

Notes: The figure shows that $5 \%$ of the 39 STR users were resistant to zidovudine, $23 \%$ to tenofovir disoproxil fumarate, and $10 \%$ were resistant to atazanavir/ritonavir or lopinavir/ritonavir. Of the patients who failed Complera or Triumeq treatment, $38 \%$ and $33 \%$ were resistant to atazanavir/ritonavir or lopinavir/ritonavir, respectively. Abbreviations: INSTI, integrase strand transfer inhibitor; NRTI, nucleoside reverse transcriptase inhibitor; NNRTI, non-nucleoside reverse transcriptase inhibitor; PI, protease inhibitor; STR, single-tablet regimen.

drugs (Figure 3). The most common NRTI drug resistance-associated mutations were M184V/I (71.8\%) and K65R (17.9\%). For NNRTI, the most common drug resistance-associated mutations were K103N (35.9\%),
V179D (33.3\%), Y181C (12.8\%), and L100I (12.8\%), while for PI, they were K20T (7.7\%) and L90M (5.1\%). One of the three patients (33.3\%) who failed Triumeq treatment had E138K, G140S, and Q148H INSTI mutations (Figure 4). The 
detailed clinical features for five STR users without drug resistance are shown in Table 2. Four of five STR users were subtype B and one was CRF07_BC. The reason why 5 STR users did not have resistance when failure was probably due to the low sensitivity for GRT in detection of resistance and poor patients adherence with emergence of wild type virus when discontinuation of HAART.
There were no associations of CD4, viral load, HIV subtype, months on current regimens, and prevalence of drug resistance between those patients being treatment naïve and initiated STR and the patients failing treatment after switching to an STR (Table 3). We could not identify the risk factors for any class of antiretroviral drug resistance. Only $23 \%$ of the patients were resistant to TDF; we further analyzed the

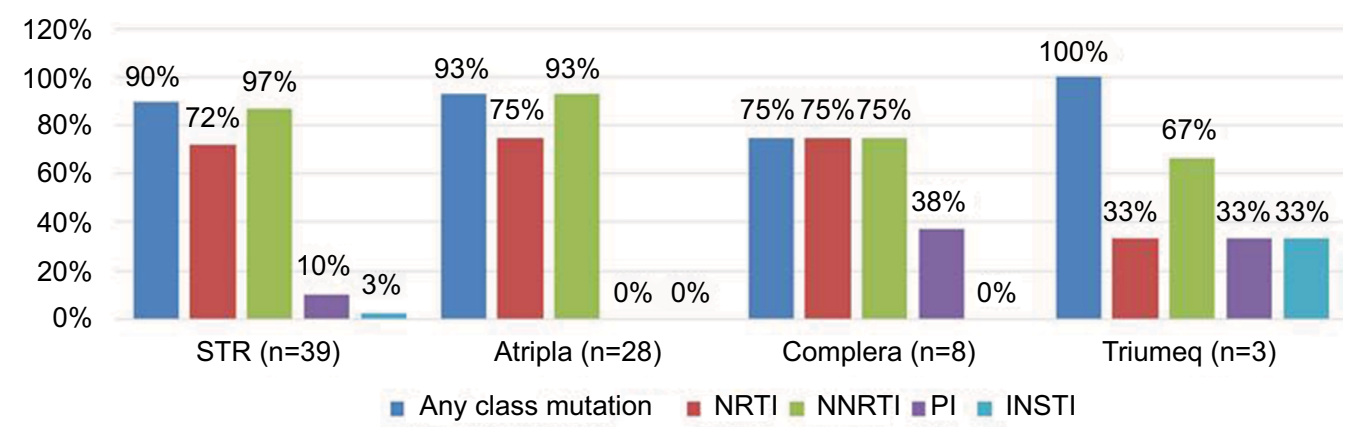

Figure 3 Percentage of HIV drug resistance-associated mutations to NRTI, NNRTI, PI, and INSTI among the 39 HIV-I-infected patients with virological failure to STRs. Abbreviations: INSTI, integrase strand transfer inhibitor; NRTI, nucleoside reverse transcriptase inhibitor; NNRTI, non-nucleoside reverse transcriptase inhibitor; PI, protease inhibitor; STR, single-tablet regimen.
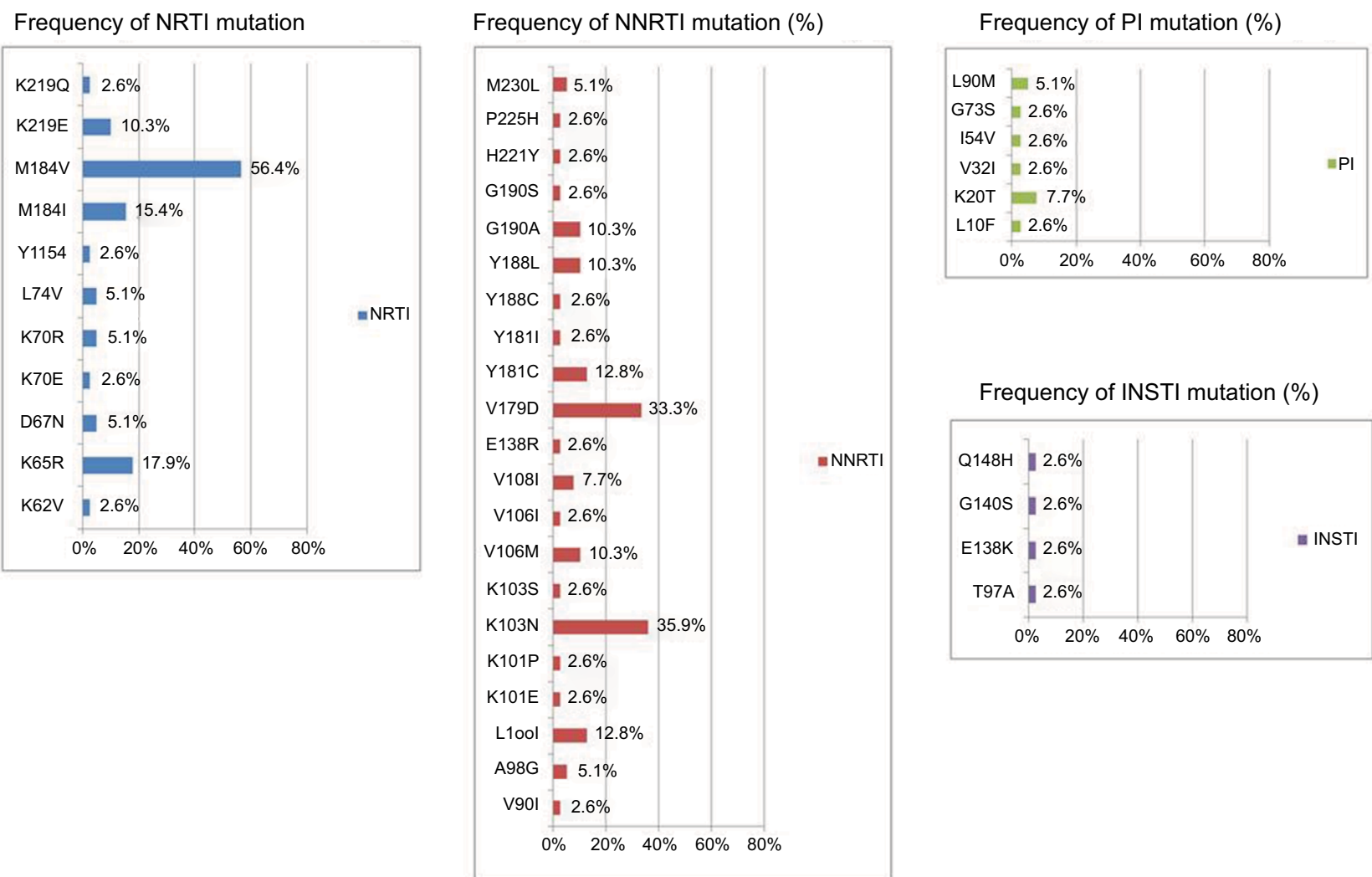

Frequency of INSTI mutation (\%)

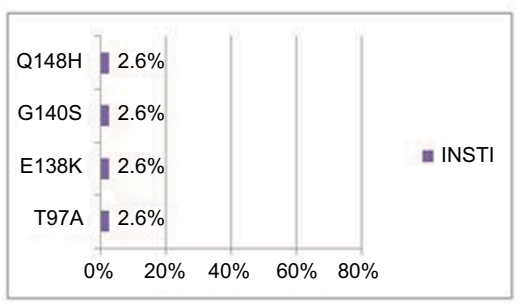

Figure 4 The prevalence of drug resistance-associated mutations to NRTI, NNRTI, PI, and INSTI among the 39 HIV-I-infected patients with virological failure to STRs. Notes: The figure shows that the most common NRTI drug resistance-associated mutations were MI84V/I (7I.8\%) and K65R (I7.9\%). For NNRTI, the mutations were KI03N (35.9\%), VI79D (33.3\%), YI8IC (I2.8\%), and LIO0I (I2.8\%), and those for PI were K20T (7.7\%) and L90M (5.I\%). One of the three patients (33.3\%) who failed Triumeq treatment had EI38K, GI40S, and QI48H INSTI mutations.

Abbreviations: INSTI, integrase strand transfer inhibitor; NRTI, nucleoside reverse transcriptase inhibitor; NNRTI, non-nucleoside reverse transcriptase inhibitor; PI, protease inhibitor; STR, single-tablet regimen. 


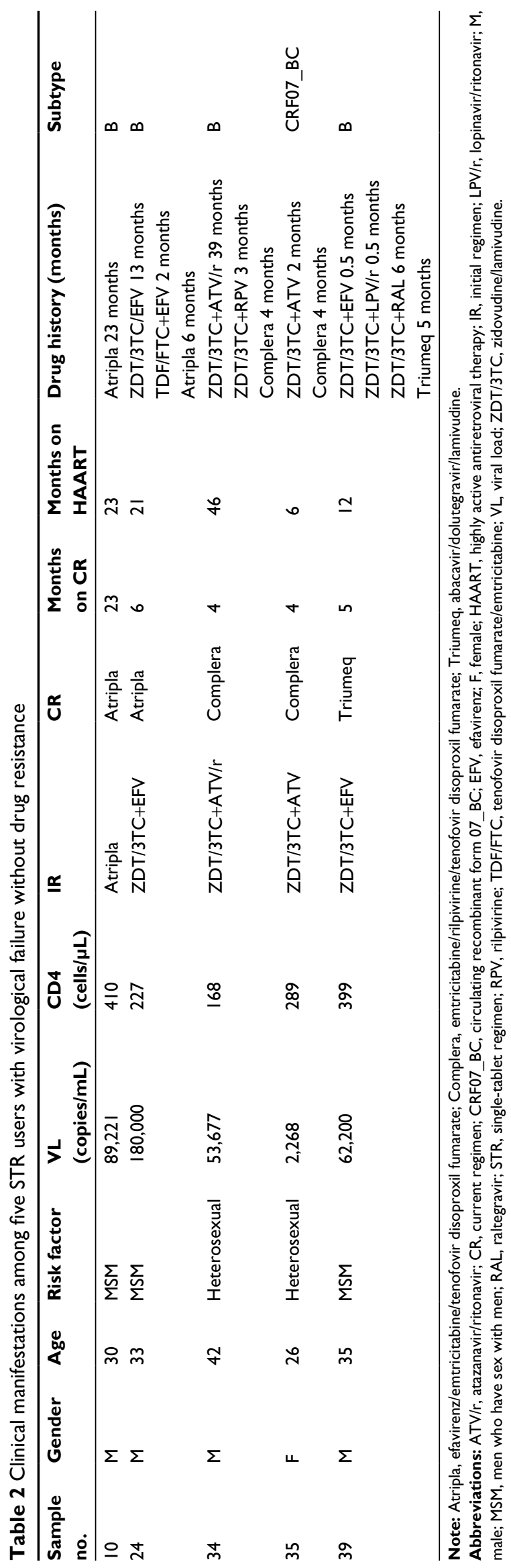

risk factor for TDF resistance. There were also no associations between TDF resistance and transmission risk factors, HIV subtypes, duration of ART and different STRs (Table 4). The patients with PI resistance were more likely to respond to treatment with a non-Atripla-based STR $(P=0.004)$ and a longer duration of ART (101 months [72.0-123.3] vs 11 months [7-44], $P=0.007$ ), as shown in Table 5.

\section{Discussion}

The current study illustrates the prevalence of HIV drug resistance among HIV-infected patients with virological failure after STR treatment in southern Taiwan. In particular, this study highlights the high rate of drug resistance (87\%) to any of the four classes of ART currently available in Taiwan. There was no difference in the prevalence of drug resistance between patients being treatment naïve and initiated STR and patients failing treatment after switching to an STR. PI resistance was associated with a non-Atripla-based STR $(P=0.004)$ and a longer duration of ART (101 months [72.0-123.3] vs 11 months [7-44], $P=0.007)$.

The risk of virological failure was low in the treatmentnaïve HIV-1-infected patients starting an STR and in HIV RNA viral-suppressed patients switching to a co-formulation of EFV/FTC/TDF or RPV/FTC/TDF. ${ }^{18,19}$ In a French study, researchers reported that virological failure occurred in 5\% (4/76) of patients starting EFV/FTC/TDF between 1 Jan 2007 and 1 June $2010 .{ }^{18}$ In an Italian study, Gagliardini et al reported that among 1,560 stable patients who switched to $\mathrm{EFV} / \mathrm{FTC} / \mathrm{TDF}$ and RPV/FTC/TDF, virological failure occurred in 44/1,097 (4\%) of those receiving EFV/FTC/TDF and 29/463 (6\%) of those receiving RPV/FTC/TDF during a maximum follow-up period of 3 years. ${ }^{19}$ Another Italian study examined the impact of pre-existing NRTI and NNRTI resistance on the maintenance of virological suppression in HIV-1-infected patients who switched to an RPV/FTC/TDF regimen. By 72 weeks, the probability of virological failure was $9.4 \%$ among the 309 patients who did not have pre-existing NRTI and NNRTI resistance. ${ }^{20}$ Taken together, although the risk of virological failure is low in patients using STRs, the prevalence of drug resistance is high in patients who failed STR treatment. In the Single-Tablet Regimen (STaR) study, the primary protocol-defined resistance analysis population included 20/394 (5.1\%) patients receiving RPV/FTC/TDF and 7/392 (1.8\%) patients receiving EFV/FTC/TDF at Week 48. In the RPV/FTC/TDF group, isolates from 17/20 (85\%) of the resistance analysis population showed the development of NNRTI and/or NRTI resistance mutations. In the EFV/FTC/TDF group, isolates from 3/7 (43\%) patients 
Table 3 Demographic information, antiretroviral treatment duration, and resistance between 8 treatment-naïve patients initiating an STR and 31 treatment-experienced patients switching to an STR with virological failure

\begin{tabular}{|c|c|c|c|c|c|}
\hline Clinical features & $\begin{array}{l}\text { Treatment naïve and } \\
\text { initiated an STR with } \\
\text { virological failure }(n=8)\end{array}$ & $\begin{array}{l}\text { Treatment experienced and } \\
\text { switched to an STR with } \\
\text { virological failure }(n=31)\end{array}$ & $P$-value & aHR & $95 \% \mathrm{Cl}$ \\
\hline \multicolumn{6}{|l|}{ Gender } \\
\hline Male & $8(100)$ & $29(93.5)$ & 1.000 & & \\
\hline Female & $0(0)$ & $2(6.5)$ & & & \\
\hline Age, median (IQR) & $29(25-38)$ & $34(27-39)$ & 0.374 & & \\
\hline \multicolumn{6}{|l|}{ Risk factor, $\mathrm{n}(\%)$} \\
\hline MSM & $8(100)$ & $26(86.7)$ & 0.560 & & \\
\hline Non-MSM & $0(0)$ & $4(13.3)$ & & & \\
\hline Viral load (log), median (IQR) & $4.7(4.0-5.1)$ & $4.7(3.9-5.2)$ & 0.972 & & \\
\hline CD4, median (IQR) & $367(163-416)$ & 255 (136-399) & 0.414 & & \\
\hline \multicolumn{6}{|l|}{ HIV subtype, n (\%) } \\
\hline B & $7(87.5)$ & $28(90.3)$ & 1.000 & 0.750 & $0.067-8.350$ \\
\hline Non-B & $\mathrm{I}(12.5)$ & $3(9.7)$ & & & \\
\hline \multicolumn{6}{|l|}{ Current regimen, n (\%) } \\
\hline Atripla & $8(100)$ & $20(64.5)$ & 0.078 & & \\
\hline Non-Atripla & $0(0)$ & II (35.5) & & & \\
\hline Months on HAART, median (IQR) & $8.5(3.3-12.5)$ & $21(9-73)$ & $0.024^{*}$ & & \\
\hline Months on CR, median (IQR) & $8.5(3.3-12.5)$ & $6(2-8)$ & 0.151 & & \\
\hline \multicolumn{6}{|l|}{ Months on CR cut-off point } \\
\hline I-6 months & $3(37.5)$ & I5 (48.4) & 0.702 & 1.563 & $0.317-7.703$ \\
\hline$\geqq 6$ months & $5(62.5)$ & $16(51.6)$ & & & \\
\hline $1-12$ months & $6(75)$ & $27(87.1)$ & 0.583 & 2.250 & $0.332-15.256$ \\
\hline$\geqq 12$ months & $2(25)$ & $4(12.9)$ & & & \\
\hline \multicolumn{6}{|l|}{ K65R mutation } \\
\hline $\mathrm{Y}$ & $2(25)$ & $5(16.1)$ & 0.617 & 1.733 & $0.269-11.187$ \\
\hline $\mathrm{N}$ & $6(75)$ & $26(83.9)$ & & & \\
\hline \multicolumn{6}{|l|}{ Any class resistance } \\
\hline$Y$ & $7(87.5)$ & $27(87.1)$ & 1.000 & 1.037 & $0.100-10.806$ \\
\hline$N$ & $\mathrm{I}(\mathrm{I} 2.5)$ & $4(12.9)$ & & & \\
\hline \multicolumn{6}{|l|}{ PI resistance } \\
\hline Y & $0(0)$ & $4(12.9)$ & 0.563 & & \\
\hline $\mathrm{N}$ & $8(100)$ & $27(87.1)$ & & & \\
\hline \multicolumn{6}{|l|}{ TDF resistance } \\
\hline Y & $2(25)$ & $7(22.6)$ & 1.000 & 1.143 & $0.187-6.971$ \\
\hline $\mathrm{N}$ & $6(75)$ & $24(77.4)$ & & & \\
\hline \multicolumn{6}{|l|}{ HBV } \\
\hline Y & $\mathrm{I}(\mathrm{I} 2.5)$ & $5(16.1)$ & 1.000 & 0.743 & $0.074-7.436$ \\
\hline $\mathrm{N}$ & $7(87.5)$ & $26(83.9)$ & & & \\
\hline
\end{tabular}

Note: Atripla, efavirenz/emtricitabine/tenofovir disoproxil fumarate; $* P<0.05$.

Abbreviations: aHR, adjusted hazard ratio; CR, current regimen; HAART, highly active antiretroviral therapy; HBV, hepatitis B virus; IQR, interquartile range; MSM, men who have sex with men; N, no; PI, protease inhibitor; STR, single-tablet regimen; TDF, tenofovir disoproxil fumarate; Y, yes.

showed the development of NNRTI and/or NRTI resistance mutations. ${ }^{21}$ These findings were similar to the results of our study, in which a high rate of drug resistance (87\%) was noted to any of the four classes of ART.

In the present study, $10 \%$ of the patients developed PI resistance. Nine of the 31 treatment-experienced patients had PI in their initial regimen and 4 developed resistance after switching to STRs. The emergence of PI resistance at the time of virological failure was uncommon in PI-naïve patients who experienced virological failure while taking a PI regimen. In previous studies in Taiwan, the reported transmitted drug resistance rate to PI was extremely low $(<4 \%) .{ }^{7,8}$ The presence of PI resistance in treatment-experienced patients who switched to an STR is interesting. In addition, $10 \%$ of the 39 STR users were resistant to atazanavir/ritonavir or lopinavir/ritonavir. In particular, $38 \%$ and $33 \%$ of those receiving Complera and Triumeq were resistant to atazanavir/ritonavir or lopinavir/ritonavir, respectively. In a Phase $3 \mathrm{~b}$, randomized, open-label, international, 48-week switch study, researchers compared the efficacy and safety of antiretroviral simplification from a ritonavir-boosted PI-based regimen to the STR (RPV/FTC/TDF) in virologically suppressed, HIV-1-infected patients. They found that no patient in the PI with two NRTIs arm met the criteria for resistance analysis after switching 
Table 4 Risk factors associated with TDF resistance in univariate analysis

\begin{tabular}{|c|c|c|c|c|c|}
\hline Clinical features & TDF resistance $(n=9)$ & Non-TDF resistance $(n=30)$ & $P$-value & aHR & $95 \% \mathrm{Cl}$ \\
\hline \multicolumn{6}{|l|}{ Gender } \\
\hline Male & $9(100)$ & $28(93.3)$ & 1.000 & & \\
\hline Female & $0(0)$ & $2(6.7)$ & & & \\
\hline Age (years), median (IQR) & $34(28-38)$ & $33(26-39)$ & 0.802 & & \\
\hline \multicolumn{6}{|l|}{ Risk factor, $\mathrm{n}(\%)$} \\
\hline MSM & $8(100)$ & $26(86.7)$ & 0.560 & & \\
\hline Non-MSM & $0(0)$ & $4(13.3)$ & & & \\
\hline Viral load (log), median (IQR) & $5.0(4.0-5.3)$ & $4.5(3.9-5.0)$ & 0.424 & & \\
\hline CD4, median (IQR) & $253(78-4 \mid 4)$ & $289(143-410)$ & 0.677 & & \\
\hline \multicolumn{6}{|l|}{ HIV subtype, n (\%) } \\
\hline B & $7(77.8)$ & $28(93.3)$ & 0.223 & 0.250 & $0.030-2.099$ \\
\hline Non-B & $2(22.2)$ & $2(6.7)$ & & & \\
\hline \multicolumn{6}{|l|}{ Current regimen, n (\%) } \\
\hline Atripla & $7(77.8)$ & $21(70)$ & 1.000 & 1.500 & $0.259-8.673$ \\
\hline Non-Atripla & $2(22.2)$ & $9(30)$ & & & \\
\hline \multicolumn{6}{|l|}{ Drug history } \\
\hline Naïve to STR & $2(22.2)$ & $6(20)$ & 1.000 & 1.143 & $0.187-6.971$ \\
\hline Change to STR & 7 (77.8) & $24(80)$ & & & \\
\hline \multicolumn{6}{|l|}{ Initial regimen, $\mathrm{n}(\%)$} \\
\hline STR & $2(22.2)$ & $6(20)$ & 1.000 & 1.143 & $0.187-6.971$ \\
\hline Non-STR & $7(77.8)$ & $24(80)$ & & & \\
\hline \multicolumn{6}{|l|}{ Non-STR of initial regimen, $n=3$ I } \\
\hline NNRTI based & $5(7 I .4)$ & $17(70.8)$ & 1.000 & 1.029 & $0.160-6.620$ \\
\hline PI based & $2(28.6)$ & $7(29.2)$ & & & \\
\hline \multicolumn{6}{|l|}{ NRTIs of initial regimen } \\
\hline ZDT/3TC & $3(33.3)$ & $19(63.3)$ & & & \\
\hline $\mathrm{TDF} / 3 \mathrm{TC}$ & I (II.I) & I (3.3) & & & \\
\hline TDF/FTC & $\mathrm{I}(\mathrm{II} .1)$ & $2(6.7)$ & & & \\
\hline Others & $4(44.5)$ & $8(26.7)$ & & & \\
\hline \multicolumn{6}{|l|}{ NNRTIs of initial regimen, $n=24$} \\
\hline EFV & $2(40)$ & I4 (82.3) & & & \\
\hline NVP & $3(60)$ & I (5.9) & & & \\
\hline RPV & $0(0)$ & $2(11.8)$ & & & \\
\hline \multicolumn{6}{|l|}{ Pls of initial regimen, $n=9$} \\
\hline Boosted-PI & $\mathrm{I}(50)$ & $5(7 \mid .4)$ & 1.000 & 0.400 & $0.016-10.017$ \\
\hline Unboosted-PI & I (50) & $2(28.6)$ & & & \\
\hline Months on HAART, median (IQR) & $45(6.0-95.5)$ & $12.5(8.0-44.5)$ & 0.526 & & \\
\hline Months on current regimen, median (IQR) & $6.0(1.5-11.5)$ & $6.0(2.8-8.3)$ & 0.920 & & \\
\hline \multicolumn{6}{|l|}{ Months on current regimen cut-off point } \\
\hline I-6 months & $4(44.4)$ & 14 (46.7) & 1.000 & 1.094 & $0.245-4.891$ \\
\hline$\geqq 6$ months & $5(55.6)$ & $16(53.3)$ & & & \\
\hline $1-12$ months & $7(77.8)$ & $26(86.7)$ & 0.607 & 1.857 & $0.280-12.311$ \\
\hline$\geqq 12$ months & $2(22.2)$ & $4(13.3)$ & & & \\
\hline \multicolumn{6}{|l|}{ Any class resistance } \\
\hline$Y^{\prime}$ & $9(100)$ & $25(83.3)$ & 0.318 & & \\
\hline $\mathrm{N}$ & $0(0)$ & $5(16.7)$ & & & \\
\hline \multicolumn{6}{|l|}{ PI resistance } \\
\hline Y & $2(22.2)$ & $2(6.7)$ & 0.223 & 4.000 & $0.476-33.585$ \\
\hline $\mathrm{N}$ & $7(77.8)$ & $28(93.3)$ & & & \\
\hline \multicolumn{6}{|l|}{ HBV } \\
\hline Y & $2(22.2)$ & $4(13.3)$ & 0.607 & 1.857 & $0.280-12.311$ \\
\hline $\mathrm{N}$ & $7(77.8)$ & $26(86.7)$ & & & \\
\hline
\end{tabular}

Notes: Pls in the initial regimen. For patients who developed TDF resistance, the initial regimen of boosted-PI at failure consisted of lopinavir/ritonavir ( $\mathrm{n}=\mathrm{I}$ ). For patients who developed TDF resistance, the initial regimen of unboosted-PI at failure consisted of atazanavir $(n=I)$. For patients who did not develop TDF resistance, the initial regimen of boosted-PI at failure consisted of lopinavir/ritonavir $(n=4)$ and atazanavir/ritonavir $(n=1)$. For patients who did not develop TDF resistance, the initial regimen of unboosted-PI at failure consisted of atazanavir $(\mathrm{n}=2)$. Atripla, efavirenz/emtricitabine/tenofovir disoproxil fumarate; Complera, emtricitabine/rilpivirine/tenofovir disoproxil fumarate; Triumeq, abacavir/dolutegravir/lamivudine.

Abbreviations: aHR, adjusted hazard ratio; EFV, efavirenz; HAART, highly active antiretroviral therapy; HBV, hepatitis B virus; IDU, intravenous drug abuser; IQR, interquartile range; MSM, men who have sex with men; N, no; NNRTI, non-nucleoside reverse transcriptase inhibitor; NRTI, nucleoside reverse transcriptase inhibitor; NVP, nevirapine; PI, protease inhibitor; RPV, rilpivirine; STR, single-tablet regimen; TDF/3TC, tenofovir disoproxil fumarate/lamivudine; TDF/FTC, tenofovir disoproxil fumarate/ emtricitabine; Y, yes; ZDT/3TC, zidovudine/lamivudine. 
Table 5 Risk factors associated with PI resistance among the 39 patients with STR failure

\begin{tabular}{|c|c|c|c|c|c|}
\hline Clinical features & PI resistance $(n=4)$ & Non-PI resistance $(n=35)$ & $P$-value & aHR & $95 \% \mathrm{Cl}$ \\
\hline \multicolumn{6}{|l|}{ Gender } \\
\hline Male & $4(100)$ & $33(94.3)$ & 1.000 & & \\
\hline Female & $0(0)$ & $2(5.7)$ & & & \\
\hline Age (years), median (IQR) & $44(33-58)$ & $33(26-38)$ & 0.078 & & \\
\hline \multicolumn{6}{|l|}{ Risk factor, n (\%) } \\
\hline MSM & $3(100)$ & $31(88.6)$ & 1.000 & & \\
\hline Non-MSM & $0(0)$ & $4(11.4)$ & & & \\
\hline Viral load (log), median (IQR) & $4.7(3.9-5.3)$ & $4.7(3.9-5.1)$ & 0.853 & & \\
\hline CD4, median (IQR) & $95(6-432)$ & $289(\mid 45-410)$ & 0.229 & & \\
\hline \multicolumn{6}{|l|}{ HIV subtype, n (\%) } \\
\hline $\mathrm{B}$ & $3(75)$ & $32(91.4)$ & 0.363 & $0.28 \mathrm{I}$ & $0.022-3.616$ \\
\hline Non-B & $\mathrm{I}(25)$ & $3(8.6)$ & & & \\
\hline \multicolumn{6}{|l|}{ Current regimen, $\mathrm{n}(\%)$} \\
\hline Atripla & $0(0)$ & $28(80)$ & $0.004^{*}$ & & \\
\hline Non-Atripla & $4(100)$ & $7(20)$ & & & \\
\hline \multicolumn{6}{|l|}{ Drug history } \\
\hline Naïve to STR & $0(0)$ & $8(22.9)$ & 0.563 & & \\
\hline Change to STR & $4(100)$ & $27(77.1)$ & & & \\
\hline \multicolumn{6}{|l|}{ Initial regimen, $\mathrm{n}(\%)$} \\
\hline STR & $0(0)$ & $8(22.9)$ & 0.563 & & \\
\hline Non-STR & $4(100)$ & $27(77.1)$ & & & \\
\hline \multicolumn{6}{|l|}{ Non-STR of initial regimen, $n=3$ I } \\
\hline NNRTI based & $\mathrm{I}(25)$ & $21(77.8)$ & 0.063 & 0.095 & $0.008-1.091$ \\
\hline PI based & $3(75)$ & $6(22.2)$ & & & \\
\hline \multicolumn{6}{|l|}{ NRTIs of initial regimen } \\
\hline ZDT/3TC & $2(50)$ & $20(57.1)$ & & & \\
\hline TDF/3TC & $0(0)$ & $2(5.7)$ & & & \\
\hline TDF/FTC & $0(0)$ & $3(8.6)$ & & & \\
\hline Others & $2(50)$ & $10(28.6)$ & & & \\
\hline \multicolumn{6}{|l|}{ NNRTls of initial regimen, $n=22$} \\
\hline EFV & $I(100)$ & $15(7 \mid .5)$ & & & \\
\hline NVP & $0(0)$ & $4(19.0)$ & & & \\
\hline RPV & $0(0)$ & $2(9.5)$ & & & \\
\hline \multicolumn{6}{|l|}{ Pls of initial regimen } \\
\hline Boosted-PI & $2(66.7)$ & $4(66.7)$ & 1.000 & 1.000 & $0.053-18.915$ \\
\hline Unboosted-PI & I (33.3) & $2(33.3)$ & & & \\
\hline Months on HAART, median (IQR) & $101(72.0-123.3)$ & II (7-44) & $0.007 *$ & & \\
\hline Months on CR, median (IQR) & $2(1.3-5.8)$ & $6(3-10)$ & 0.124 & & \\
\hline \multicolumn{6}{|l|}{ Months on CR cut-off point } \\
\hline I-6 months & $3(75)$ & $15(42.9)$ & 0.318 & 0.250 & $0.024-2.648$ \\
\hline$\geqq 6$ months & $I(25)$ & $20(57.1)$ & & & \\
\hline $1-12$ months & $4(100)$ & $29(82.9)$ & 1.000 & & \\
\hline$\geqq 12$ months & $0(0)$ & $6(17.1)$ & & & \\
\hline \multicolumn{6}{|l|}{ K65R mutation } \\
\hline Y & $\mathrm{I}(25)$ & $6(17.1)$ & 0.563 & 1.611 & $0.142-18.262$ \\
\hline $\mathrm{N}$ & $3(75)$ & $29(82.9)$ & & & \\
\hline \multicolumn{6}{|l|}{ Any class resistance } \\
\hline$Y$ & $4(100)$ & $30(85.7)$ & 1.000 & & \\
\hline $\mathrm{N}$ & $0(0)$ & $5(14.3)$ & & & \\
\hline \multicolumn{6}{|l|}{ TDF resistance } \\
\hline Y & $2(50)$ & $7(20)$ & 0.223 & 4.000 & $0.476-33.585$ \\
\hline$N$ & $2(50)$ & $28(80)$ & & & \\
\hline \multicolumn{6}{|l|}{ HBV } \\
\hline Y & I (25) & $5(14.3)$ & 0.502 & 2.000 & $0.172-23.251$ \\
\hline $\mathrm{N}$ & $3(75)$ & $30(85.7)$ & & & \\
\hline
\end{tabular}

Note: Atripla, efavirenz/emtricitabine/tenofovir disoproxil fumarate; Complera, emtricitabine/rilpivirine/tenofovir disoproxil fumarate; Triumeq, abacavir/dolutegravir/lamivudine; $* P<0.05$.

Abbreviations: aHR, adjusted hazard ratio; CR, current regimen; EFV, efavirenz; HAART, highly active antiretroviral therapy; HBV, hepatitis B virus; IQR, interquartile range; MSM, men who have sex with men; N, no; NNRTI, non-nucleoside reverse transcriptase inhibitor; NRTI, nucleoside reverse transcriptase inhibitor; NVP, nevirapine; $\mathrm{PI}$, protease inhibitor; RPV, rilpivirine; STR, single-tablet regimen; TDF/3TC, tenofovir disoproxil fumarate/lamivudine; TDF/FTC, tenofovir disoproxil fumarate/emtricitabine; Y, yes; ZDT/3TC, zidovudine/lamivudine. 
to RPV/FTC/TDF through Week $48 .^{22}$ The reason why PI resistance was being observed in those treatment-experienced patients was probably due to the inappropriate switching from a multiple tablet regimen to an STR without guidance by a GRT. The principle for switching a HAART regimen in the Department of Health and Human Services guidelines is based on a review of ART history, drug resistance test results before switching, and increased monitoring during the first 3 months after switching. Care is needed when switching from a booster PI to another class if full treatment or resistance history is not known. ${ }^{23}$ This should remind clinicians that detailed drug resistance history and close monitoring are mandatory after switching to an STR.

\section{Limitations}

The sample size was small, and only $8 / 39$ patients were treatment naïve and failed STR treatment. The prevalence rate for STR failure could not be calculated due to the lack of data on the total number of STR users who did not harbor resistance. In addition, data on the baseline prevalence of HIV-transmitted drug resistance for the population studied were also unavailable. Routine GRT in treatment-naïve HIV-1-infected patients is not reimbursed by the health insurance system or the government, although the prevalence of transmitted drug resistance has been reported to be around $8 \%-10 \%$ in Taiwan. ${ }^{7,8}$ In addition, the duration of virological failure before GRT was not precisely determined, even though routine viral load monitoring is available in Taiwan. The doctors in-charge may have had limited access to the GRT due to non-reimbursement from the National Health Insurance System. Although GRT has been found to have a significant benefit on the virological response when choosing the salvage regimens in patients failing ART, ${ }^{24-26}$ the treatment outcomes for patients with virological failure after switching to another regimen are not available, making it impossible to evaluate the clinical impact of GRT in these patients. Data were also lacking on adherence to the ART regimen. Lastly, the results were from southern Taiwan with predominantly MSM subtype B patients. Thus, it would be interesting to extend this work to other risk groups or other areas of Taiwan.

\section{Conclusion}

This study showed a high rate of antiretroviral drug resistance in patients who failed STR treatment. The presence of PI resistance in those who failed STR treatment was unexpected, and it represents an inappropriate switch in drug regimen from a multiple tablet regimen to an STR. This should remind the clinician that a detailed drug resistance history and close monitoring are mandatory after switching to an STR.

\section{Acknowledgments}

The authors thank Miss Pei-Yun Chou, Department of Infectious Diseases, Kaohsiung Veterans General Hospital, Taiwan for laboratory technical support. This study was funded by the Medical Foundation in Memory of Dr DehLin Cheng (MF-DLC100053S5C2Y1) and by the Veterans General Hospitals and University System of Taiwan Joint Research Program grants (VGHUST105-G3-2-1 and VGHUST106-G3-1-1).

\section{Disclosure}

The authors report no conflicts of interest in this work.

\section{References}

1. Sterne JA, Hernán MA, Ledergerber B, et al. Long-term effectiveness of potent antiretroviral therapy in preventing AIDS and death: a prospective cohort study. Lancet. 2005;366(9483):378-384.

2. Bor J, Herbst AJ, Newell ML, Bärnighausen T. Increases in adult life expectancy in rural South Africa: valuing the scale-up of HIV treatment. Science. 2013;339(6122):961-965.

3. Jin Y, Liu Z, Wang X, et al. A systematic review of cohort studies of the quality of life in HIV/AIDS patients after antiretroviral therapy. Int J STD AIDS. 2014;25(11):771-777.

4. Kumarasamy N, Solomon S, Chaguturu SK, et al. The changing natural history of HIV disease: before and after the introduction of generic antiretroviral therapy in southern India. Clin Infect Dis. 2005;41(10): $1525-1528$.

5. Taniguchi T, Nurutdinova D, Grubb JR, et al. Transmitted drug-resistant HIV type 1 remains prevalent and impacts virologic outcomes despite genotype-guided antiretroviral therapy. AIDS Res Hum Retroviruses. 2012;28(3):259-264.

6. Rupérez M, Pou C, Maculuve S, et al. Determinants of virological failure and antiretroviral drug resistance in Mozambique. J Antimicrob Chemother. 2015;70(9):2639-2647.

7. Lai CC, Liu WC, Fang CT, et al. Transmitted drug resistance of HIV-1 strains among individuals attending voluntary counselling and testing in Taiwan. J Antimicrob Chemother. 2016;71(1):226-234.

8. Weng YW, Tsai HC, Lee SS, et al. Prevalence and associated factors for HIV-1 transmitted drug resistance in voluntary clients for counseling and testing in Southern Taiwan. J Microbiol Immunol Infect. 2016;49(4):487-493.

9. Tsai HC, Chen IT, Wu KS, et al. High rate of HIV-1 drug resistance in treatment failure patients in Taiwan, 2009-2014. Infect Drug Resist. 2017;10:343-352.

10. Centers of Diseases Control Taiwan.: Statistics of HIV/AIDS; 2017. Available from: https://www.cdc.gov.tw/english/submenu.aspx?nowtr eeid=F6F562FD95FD8DF9\&treeid=00ED75D6C887BB27. Accessed January 27, 2018.

11. Bangsberg DR, Ragland K, Monk A, Deeks SG. A single tablet regimen is associated with higher adherence and viral suppression than multiple tablet regimens in HIV+ homeless and marginally housed people. AIDS. 2010;24(18):2835-2840.

12. Airoldi M, Zaccarelli M, Bisi L, et al. One-pill once-a-day HAART: a simplification strategy that improves adherence and quality of life of HIV-infected subjects. Patient Prefer Adherence. 2010;4:115-125.

13. Blanco JL, Montaner JS, Marconi VC, et al. Lower prevalence of drug resistance mutations at first-line virological failure to first-line therapy with atripla vs. tenofovir + emtricitabine/lamivudine + efavirenz administered on a multiple tablet therapy. AIDS. 2014;28(17):2531-2539.

14. Cotte L, Ferry T, Pugliese P, et al. Effectiveness and tolerance of single tablet versus once daily multiple tablet regimens as first-line antiretroviral therapy - Results from a large french multicenter cohort study. PLoS One. 2017;12(2):e0170661. 
15. Canducci F, Barda B, Ceresola E, et al. Evolution patterns of raltegravirresistant mutations after integrase inhibitor interruption. Clin Microbiol Infect. 2011;17(6):928-934.

16. Canducci F, Ceresola ER, Boeri E, et al. Cross-resistance profile of the novel integrase inhibitor Dolutegravir (S/GSK1349572) using clonal viral variants selected in patients failing raltegravir. J Infect Dis. 2011;204(11): 1811-1815.

17. Wensing AM, Calvez V, Günthard HF, et al. Update of the Drug Resistance Mutations in HIV-1. Top Antivir Med. 2017;2017(24): 132-133.

18. Gallien S, Flandre P, Nguyen N, et al. Safety and efficacy of coformulated efavirenz/emtricitabine/tenofovir single-tablet regimen in treatmentnaive patients infected with HIV-1. J Med Virol. 2015;87(2):187-191.

19. Gagliardini R, Bandera A, Zaccarelli M, et al. 3-Year efficacy and durability of simplification to single tablet regimens: a comparison between co-formulated efavirenz/emtricitabine/tenofovir and rilpivirine/ emtricitabine/tenofovir. Antivir Ther. 2018;23:139-148.

20. Armenia D, di Carlo D, Calcagno A, et al. Pre-existent NRTI and NNRTI resistance impacts on maintenance of virological suppression in HIV-1-infected patients who switch to a tenofovir/emtricitabine/ rilpivirine single-tablet regimen. JAntimicrob Chemother. 2017;72(3): $855-865$
21. Porter DP, Kulkarni R, Fralich T, Miller MD, White KL. Characterization of HIV-1 drug resistance development through week 48 in antiretroviral naive subjects on rilpivirine/emtricitabine/tenofovir DF or efavirenz/ emtricitabine/tenofovir DF in the STaR study (GS-US-264-0110. J Acquir Immune Defic Syndr. 2014;65(3):318-326.

22. Palella FJ, Fisher M, Tebas P, et al. Simplification to rilpivirine/emtricitabine/tenofovir disoproxil fumarate from ritonavir-boosted protease inhibitor antiretroviral therapy in a randomized trial of HIV-1 RNAsuppressed participants. AIDS. 2014;28(3):335-344.

23. DHHS Panel on Antiretroviral Guidelines for Adults and Adolescents. Guidelines for the Use of Antiretroviral Agents in HIV-1-Infected Adults and Adolescents. Updated October 17, 2017.

24. Durant J, Clevenbergh P, Halfon P, et al. Drug-resistance genotyping in HIV-1 therapy: the VIRADAPT randomised controlled trial. Lancet 1999;353(9171):2195-2199.

25. Baxter JD, Mayers DL, Wentworth DN, et al. A randomized study of antiretroviral management based on plasma genotypic antiretroviral resistance testing in patients failing therapy. CPCRA 046 Study Team for the Terry Beirn Community Programs for Clinical Research on AIDS. AIDS. 2000;14(9):F83-F93.

26. Falloon J. Time to genotype for selection of antiretroviral regimens in previously treated patients? Lancet. 1999;353(9171):2173-2174.
Infection and Drug Resistance

\section{Publish your work in this journal}

Infection and Drug Resistance is an international, peer-reviewed openaccess journal that focuses on the optimal treatment of infection (bacterial, fungal and viral) and the development and institution of preventive strategies to minimize the development and spread of resistance. The journal is specifically concerned with the epidemiology of antibiotic

\section{Dovepress}

resistance and the mechanisms of resistance development and diffusion in both hospitals and the community. The manuscript management system is completely online and includes a very quick and fair peerreview system, which is all easy to use. Visit http://www.dovepress.com/ testimonials.php to read real quotes from published authors. 